\title{
Efficient Large-Scale Coating Microstructure Formation Using Realistic CFD Models
}

\author{
Thomas Wiederkehr and Heinrich Müller
}

(Submitted March 12, 2014; in revised form July 1, 2014)

\begin{abstract}
For the understanding of physical effects during the formation of thermally sprayed coating layers and the deduction of the macroscopic properties of a coating, microstructure modeling and simulation techniques play an important role. In this contribution, a coupled simulation framework consisting of a detailed, CFD-based single splat simulation, and a large-scale coating build-up simulation is presented that is capable to compute large-scale, three-dimensional, porous microstructures by sequential drop impingement of more than 10,000 individual particles on multicore workstation hardware. Due to the geometry-based coupling of the two simulations, the deformation, cooling, and solidification of every particle is sensitive to the hit surface area and thereby pores develop naturally in the model. The single splat simulation employs the highly parallel Lattice-Boltzmann method, which is well suited for GPUacceleration. In order to save splat calculations, the coating simulation includes a database-driven approach that re-uses already computed splats for similar underground shapes at the randomly chosen impact sites. For a fast database search, three different methods of efficient pre-selection of candidates are described and compared against each other.
\end{abstract}

Keywords 3D, lattice boltzmann method, microstructure formation, particle impact simulation

\section{Introduction}

In thermal spraying, the feedstock material provided as rods, wires, or powders is melted and resulting liquid material particles are accelerated toward a surface to be coated. Upon impact, the particles flatten, rapidly cool down, and solidify on top of each other thereby forming a complex coating microstructure. Due to entrapped gas in between the solidified splats a complex porous microstructure develops over time. Geometric models of such thermally sprayed microstructures are used in a wide variety of simulations to predict different physical properties of the manufactured workpieces that are relevant for the application at hand. Examples include the investigation of residual stress distributions, elastic modulus, fracture toughness, erosion resistance, or heat transfer for thermal barrier coatings (Ref 1-4).

In practice, obtaining adequately complex and realistic three-dimensional microstructure models is a difficult and time-consuming task. In this work, a novel, generic, computationally efficient simulation concept is presented that is suited to be combined with different single-particle simulations providing three-dimensional splat shapes to construct the whole coating. The concept is found on the

Thomas Wiederkehr and Heinrich Müller, Technische Universität Dortmund, Informatik VII - Graphische Systeme, Dortmund, Germany. Contact e-mail: wiederkehr@ls7.cs.tudortmund.de.

finding that single splat formation algorithms have been extensively studied in the literature in the last years, but only few publications mention "stacking" of more than three droplets not to speak of hundreds or thousands. Of course, one obvious reason for this lack of publications is limited computation power and time, since complex models consume a lot of these for one splat already. However, since macroscopic coating properties cannot reliably be deduced from just a few splats interacting, a part of the computational power has to be spent for the enlargement of the simulation domain rather than for more complex single-particle models at some point.

The key idea of the new coating simulation concept is to re-use already computed splats for similar underground shapes at the randomly chosen impact sites, in order to save time-consuming calls of the single-particle simulation. For that purpose, a database of splat configurations is built up. A splat configuration consists of an underground and the splat delivered by simulating a droplet impinging on that underground. Three different database filters are presented which allow for an efficient pre-selection of candidates in the data base with a similar underground as the query underground of a current droplet during coating simulation. The filters are inspired by methods applied in image retrieval.

A second topic of this paper is the single splat simulation. As already mentioned, several approaches have been published in the past reaching from heuristic, measurement-based, empirical methods to computational fluid dynamics (CFD) approaches. CFD approaches are usually based on the finite element (FE) method and are often time-consuming. An alternative is the Lattice-Boltzmann (LB) method which has not yet found much interest in thermal spraying. An advantage of the LB method is that it is highly parallel and that it can be immediately 
GPU-accelerated. In this paper, the thermal LB model by Yuan and Schaefer (Ref 5,6) is adopted for single splat simulation. While the presented single-particle impact model may not be as sophisticated as other state of the art droplet models it is a fully three-dimensional model including spreading, cooling, and solidification and can therefore react to any underlying substrate shape as opposed to other models currently found in most microstructure build-up simulations.

The issue of computational efficiency addressed in this paper is crucial to increase the computational domain in order to predict macroscopic effects of relevance for practical applications of coated surfaces. An example is the benefit of becoming able to predict coating porosity distributions or derived homogenous coating properties based on the local shape or curvature of the workpiece surface and the time-dependent spray torch movement using (relatively) accurate single splat models.

In the following section, an overview over related work dealing with microstructure formation by handling more than a few (say five) splats is given and some common concepts are pointed out. Section 3 briefly describes the CFD simulation implemented, which is a Lattice-Boltzmann type simulation. Section 4 covers the main part of the work describing the simulation concept as well as three examined algorithms used to locate appropriate splat shapes that have already been computed in the splat database. Section 5 presents some simulated examples and compares the mentioned splat selection algorithms.

\section{Related Work}

The simulation of single droplet impact has been extensively studied focusing on different materials, processes, and modeling techniques and will thus not be discussed here. Fauchais et al. (Ref 7), Chandra and Fauchais (Ref 8), Yang et al. (Ref 9) as well as Solonenko et al. (Ref 10) provide good surveys of the area of single-particle impact.

Work related to the development of porous microstructure models found in the literature can be categorized by the generation method into real-image-based, synthetic, and simulated meshes.

Real-image-based methods use segmentations of scanning electron microscopy or light optical microscopy images to derive meshes based on a pixel based approach (Ref 11-13). Three-dimensional meshes based on real specimens can be realized by using voxel volumes instead of single images, which may for example be derived from a series of parallel cross-sectional images as demonstrated earlier by us (Ref 14) and also Chawla et al. (Ref 15). Bobzin et al. generate 3D-voxel volumes by combining randomly selected windows of a single cross-sectional image instead of using multiple cross-sectional images, which may be more representative for the computation of homogenization properties and eliminates the need for the majority of grinding and polishing steps (Ref 16). A standard method to convert segmented voxel volumes to the required boundary representation is given by the marching cubes algorithm or enhancements thereof (Ref 17, 18).

In contrast to real-image-based methods, synthetic methods for the generation of porous volume representations describe pore- or material-distributions using stochastic and geometric methods (Ref 19). The modeled distributions are usually based on experimentally obtained data and may comprise amongst others diameter, elongation, orientation, and volume fraction information (Ref 4, 20, 21). Based on these distributions, volumes are randomly filled with pores of different types (globular, interlamellar, cracks). A slightly different approach combining real-data with synthetic methods has been chosen by Singh, who extracts real pore shapes from high-resolution cross-sectional images and copies them into the target domain while preserving statistical measures typical for synthetic methods (Ref 22).

The last method to obtain microstructure models, and to which the method presented in this paper can be attributed to, is by repeated single-particle simulation. The main concept is to successively simulate spray droplets impinging on each other, which then solidify and form the coating possibly forming pores in between. This approach is somewhat complementary to the synthetic approach in the sense that it focuses on the modeling of (solid) splats rather than on the modeling of pores.* Compared to the two other approaches, literature dealing with multi-particle simulations for the formation of complex coating microstructures is relatively sparse.

Early models simulated microstructure build-up within cross-sectional simulation domains. For example, Cirolini et al. presented a simple two-dimensional model using a physically based rule-set to construct the coating (Ref 23). Although two-dimensional models still have their uses, for example in two-dimensional homogenization problems, they are not able to capture splat formation effects like spreading around obstacles or the possibly resulting splashing or fingering. Mostaghimi et al. presented a threedimensional approach modeling the domain as a voxel (volume element) model with adaptive resolution to enhance the accuracy at splat boundaries (Ref 24, 25). Splats were successively inserted on top of the already depositioned ones by calculating their diameter using a simple analytic expression and then choosing one of only four different splat prototypes based on the distance to the next closest splat. This idea is based on the observation that for a drop impinging onto another drop the resulting splat shape depends significantly on the distance of the distance from the splat centers-at least if the lower splat is nearly disk shaped. Porosity is then introduced by assuming a certain degree of curl-up of the splat boundaries. Kang et al. simulate microstructure growth on inclined substrates and determine the elongation as well as the spread factor of their splats based on curve-fitted

\footnotetext{
*Additionally, one might view the real-data based approach as "just searching the interface" completing the list of anything that can be found in a binary solid-void-representation-however, the interface is needed for any of the methods.
} 
measurements from several real splat observations in dependence of the impact angle. However, no porosity is included in their model that only tracks the height of the coating. Globular and interlamellar pores as well as intralamellar cracks were modeled by Beauvais et al. (Ref 26). In their model randomly picked splat shapes, which are geometrically derived from 50 experimental observations, are picked and inserted in a binary voxel volume moving down all voxels individually until they hit previously inserted splats. The different types of voids are generated in three post-processing steps placing spherical globular pores, vertical intralamellar cracks as well as interlamellar pores in between splats until the desired porosity level is reached. Thus, their model is a mixture of splat stacking and synthetic pore generation.

In general, microstructures are commonly modeled using some form of voxel model, binary or with volume fractions, regular, or partially adaptive. In many cases, splats are modeled using basic geometries like (elongated) disks, spheres, or tori approximating a set of observed particle impacts and are inserted at random locations in the model. Although their shape may be adapted to parameters like temperature or impact angle drawn from probability distributions fitted to process-specific measurements, the insertion in the model frequently does not take the detailed structure of the underground the particle is deposited on into account (Ref 27). This is still a major drawback, since the development of realistic voids clearly depends on the underground geometry.

\section{Droplet Model}

The droplet model computes a single droplet impinging onto the substrate surface. Its main input is a heightmap describing the local underground shape the drop impacts upon and its main output is a geometric description of the resulting solidified splat geometry by means of a voxel based, three-dimensional density field. Because of the simplicity of this programmatic interface, various droplet simulations found in the current literature could be used to compute the splats for the coating build-up simulation described in section 4 . This includes simulations using popular level set or volume of fluid methods to describe the simulation domain, as these can be easily converted to voxel-based representations. By choosing a droplet simulation model tailored to specific processes, parameterizations, or feedstock materials, the whole coating build-up simulation can be specialized accordingly.

In this work, a multi-phase Thermal Lattice Boltzmann Model (TLBM) is used for the single droplet simulation. Two different formulations were implemented and tested (Ref 28), namely the models by Inamuro et al. (Ref 5) and by Yuan and Schaefer (Ref 6, 29). The formulation of Inamuro et al. was specifically designed to allow high density ratios of up to 1000:1 between liquid and gaseous phase, which is difficult to achieve in LB-models but is needed for many realistic simulations such as the droplet impact pursued here (Ref 30). However, the model

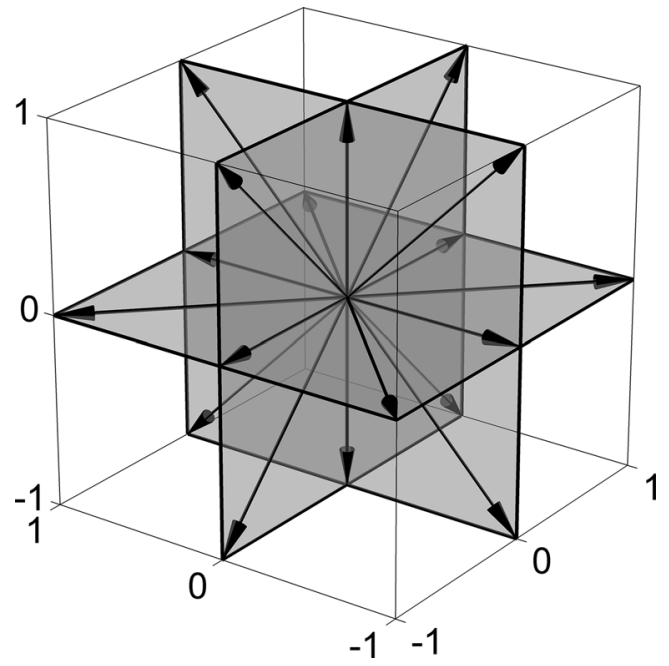

Fig. 1 Illustration of the 19 directions $e_{\alpha}$ of the D3Q19 lattice model

introduces a new equation for the correction of interfacial velocities termed "pressure correction" that needs to be solved in every time step and significantly increases the computation complexity and time. Therefore, the faster method developed by Yuan and Schaefer has been used for the results in this work. Their model is based on the well-known single component multi-phase model by Shan and Chen (Ref 31, 32) and extends it with a temperature field following the passive scalar approach that is coupled to the fluid dynamic model through a body force term. Furthermore, for this work, an indirect coupling between temperature and dynamic model is introduced by the (partial) solidification of lattice cells described later.

In the following, the basics of the model by Yuan and Schaefer, termed YSM, are described only in so far as needed to explain the modifications introduced and to give a basic understanding of the general approach of the LBM.

\subsection{Basic Model}

LB-models use a particle distribution function $f_{\alpha}(\boldsymbol{x}, t)$ to describe direction-dependent densities at position $\boldsymbol{x}$ and time $t$ moving through a macroscopic regular lattice. The lattice consists of discrete cells, $x \in V_{D} \subset Z^{3}$. The parameter $\alpha=0, \ldots, N$ specifies a discrete velocity $\boldsymbol{e}_{\alpha}$ pointing to a neighboring cell, i.e., the $\boldsymbol{e}_{\alpha}$ are of the form $(i, j, k)^{\mathrm{T}}$ with $i, j, k \in\{-1,0,1\}$. Usually only a subset of the 27 possible directions is used. The YSM employs a D3Q19 model based on the $N+1=19$ directions with $\vartheta_{i j k}=|i|+|j|+|k| \leq 2$ shown in Fig. 1. For each cell $\boldsymbol{x}$, the macroscopic mass density $\rho(\boldsymbol{x}, t)$ and velocity $\boldsymbol{u}(\boldsymbol{x}, t)$ can be computed from the particle distribution function (PDF) by

$\rho(\boldsymbol{x}, t)=\sum_{\alpha=0}^{N} f_{\alpha}(\boldsymbol{x}, t)$

and 
$\boldsymbol{u}(\boldsymbol{x}, t)=\frac{1}{\rho(\boldsymbol{x}, t)} \sum_{\alpha=0}^{N} f_{\alpha}(\boldsymbol{x}, t) \boldsymbol{e}_{\alpha}$.

The development of the PDF in one time step $\delta t$ for each cell can be expressed as

$\underbrace{f_{\alpha}\left(\boldsymbol{x}+\boldsymbol{e}_{\alpha} \delta t, t+\delta t\right)=f_{\alpha}(\boldsymbol{x}, t)}_{\text {Streaming }} \underbrace{-\frac{1}{\tau}\left[f_{\alpha}(\boldsymbol{x}, t)-f_{\alpha}^{e q}(\boldsymbol{x}, t)\right]}_{\Omega_{\alpha}}$,

where the first part streams the particle densities in the neighboring cells according to their direction and the second term $\Omega_{\alpha}$ is called the collision operator. In this case, the well-known Bhatnagar-Gross-Krook (BGK) approximation of the collision operator is used, which is represented by a linear relaxation of the flow toward the equilibrium distribution $f_{\alpha}^{\mathrm{eq}}$ with single relaxation time $\tau$ (Ref 33). The equilibrium distribution function, which relates to the Maxwell distribution describing particle velocities in kinetic gas theory, is

$$
\begin{aligned}
f_{\alpha}^{\mathrm{eq}}(\boldsymbol{x}, t)= & \rho(\boldsymbol{x}, t) w_{\alpha}\left(1+\frac{3}{c^{2}} \boldsymbol{e}_{\alpha} \cdot \boldsymbol{u}(\boldsymbol{x}, t)\right. \\
& \left.+\frac{9}{2 c^{4}}\left(\boldsymbol{e}_{\alpha} \cdot \boldsymbol{u}(\boldsymbol{x}, t)\right)^{2}-\frac{3}{2 c^{2}} \boldsymbol{u}(\boldsymbol{x}, t) \cdot \boldsymbol{u}(\boldsymbol{x}, t)\right) .
\end{aligned}
$$

Therein, "." denotes the dot product, $c=\delta x / \delta t$ is the lattice speed and the direction-dependent weighting factor $w_{\alpha}$ equals $\frac{1}{3}$ for $\vartheta_{i j k}=0, \frac{1}{18}$ for $\vartheta_{i j k}=1$, and $\frac{1}{36}$ otherwise. The YSM extends this formulation to a two-phase system by introducing attractive interparticle forces controllable through an interaction strength parameter $g_{\mathrm{f}}$ chosen as $g_{\mathrm{f}}=-0.35$ in our implementation limiting the maximal density ratio to about 42 (Ref 29). Furthermore, similar solid-liquid interaction forces are used and general body forces like gravity can be added. All forces are incorporated using the equilibrium shifting method, i.e., they are integrated in the velocity terms of $f_{\alpha}^{\text {eq }}$. An analogous second LBM is used to simulate the temperature distribution coupled to the dynamic model via a buoyancy body force term as described in Ref 6, 29.

\subsection{Boundary Conditions and Solidification}

For the implementation, Eq 3 is usually split into the collision step

$f_{\alpha}^{*}(\boldsymbol{x}, t)=f_{\alpha}(\boldsymbol{x}, t)-\frac{1}{\tau}\left[f_{\alpha}(\boldsymbol{x}, t)-f_{\alpha}^{\mathrm{eq}}(\boldsymbol{x}, t)\right]$

and the streaming step

$f_{\alpha}\left(\boldsymbol{x}+\boldsymbol{e}_{\alpha} \delta t, t+\delta t\right)=f_{\alpha}^{*}(\boldsymbol{x}, t)$,

with $f^{*}$ denoting an intermediate PDF. The collision and streaming steps are performed alternately during the simulation process.

For solid boundary nodes, a no-slip boundary condition can be applied by implementing a bounce-back scheme that mirrors the PDF at the boundary. In the employed halfway bounce-back scheme, particle densities $f_{\alpha}$ that would be propagated into a solid wall node in direction $\boldsymbol{e}_{\alpha}$ are instead reversed during the streaming step to point into direction $\bar{\alpha}$ with $\boldsymbol{e}_{\bar{\alpha}}=-\boldsymbol{e}_{\alpha}$. Thus, the streaming step for solid boundary cells becomes

$f_{\alpha}\left(\boldsymbol{x}+\boldsymbol{e}_{\alpha} \delta t, t+\delta t\right)=f_{\bar{\alpha}}^{*}\left(\boldsymbol{x}+\boldsymbol{e}_{\alpha} \delta t, t\right)$.

In addition to this no-slip scheme, which is used for the arbitrarily shaped substrate surface boundary, an approach similar to the one presented by Semma et al. (Ref 34) has been added to support solidification of cells. The phase change of each cell takes place over a small temperature range $2 \epsilon_{T}$ and the liquid fraction in each cell is modeled by

$$
\lambda(\boldsymbol{x})=\left\{\begin{array}{lll}
0, & T(\boldsymbol{x})<T_{m}-\epsilon_{T} & \text { (solid) } \\
\left(T(\boldsymbol{x})-T_{m}+\epsilon_{T}\right) / 2 \epsilon_{T}, & T_{m}-\epsilon_{T} \leq T(\boldsymbol{x}) \leq T_{m}+\epsilon_{T} & \text { (transition) }, \\
1, & T_{m}+\epsilon_{T}<T(\boldsymbol{x}) & \text { (liquid) }
\end{array}\right.
$$

where $T_{m}$ is the melting temperature and $T(\boldsymbol{x})$ the macroscopic temperature. Note that for gaseous cells $\lambda=1$. The transition between the liquid and solid phases is modeled by linear interpolation between normal cell streaming and the halfway bounce-back scheme by replacing the streaming with

$f\left(\boldsymbol{x}+\boldsymbol{e}_{\alpha} \delta t, t+\delta t\right)=\lambda(\boldsymbol{x}) f_{\alpha}^{*}(\boldsymbol{x}, t)+(1-\lambda(\boldsymbol{x})) f_{\bar{\alpha}}^{*}\left(\boldsymbol{x}+\boldsymbol{e}_{\alpha} \delta t, t\right)$.

For $\lambda=1$, this equals the normal streaming step as given in Eq 6 and for $\lambda=0$ the result is the halfway bounce-back scheme.

\subsection{Simulation Setup}

For the use of the droplet simulation to simulate a large number of splats impinging on different substrate surfaces a file-based interface has been created. After the initialization of the simulation application, a specified job-description file containing all relevant parameters is loaded and executed accordingly. The basic setup as well as two examples of the resulting splats, are shown in Fig. 2. At first the simulation domain $V_{\mathrm{D}}$ of dimension $L_{x} \times L_{y} \times L_{z}$ is initialized. Then the substrate boundary specified as an integer-valued heightmap $H(x, y)$ is loaded and all cells $\boldsymbol{x}=(x, y, z)^{\mathrm{T}}$ with $z<H(x, y)$ are marked as boundary cells with no-slip boundary condition. The initially spherical droplet of radius $r_{\mathrm{D}}=16$ cells is placed in the middle of the $x, y$-range at a fixed offset $z_{\text {off }}$ above the highest boundary cell under the droplet, i.e.,

$z_{\text {drop }}=\max \left[H(x, y) \mid \sqrt{x^{2}+y^{2}} \leq r_{\mathrm{D}}\right]+z_{\text {off }}$.

The height of the simulation domain $V_{\mathrm{D}}$ is chosen to leave a distance of 10 between the topmost droplet cell and the upper domain boundary. Cyclic boundaries are used at all domain borders. To accelerate the droplet a constant body 
force is applied to the simulation domain. $z_{\text {off }}$ has been experimentally determined to be $z_{\text {off }}=15$ lattice units, which yields a reasonable average lattice speed of the liquid droplet of $\sim 0.22$ upon first contact with a flat substrate. Therefore, in case of a totally flat surface boundary, the size of the simulation volume is at least $80 \times 80 \times 57$, where the $x$ - and $y$-dimensions have been found to be large enough to hold the solidified splat for most substrate shapes that do contain only mild slopes. In case the coating build-up simulation detects that the solidified splat touches any border of $V_{\mathrm{D}}$ the simulation is automatically repeated with a larger volume until the splat fits. Due to a lack of accurate physical parameters for the spray material, the simulation parameters have been chosen to yield splat shapes visually agreeing with diskshaped splats occurring for example at higher substrate temperatures during plasma spray deposition of Ni splats onto stainless steel substrates (Ref 9, 35). The initial temperatures have been set to $2800 \mathrm{~K}$ for the liquid droplet and $373 \mathrm{~K}$ for the gaseous cells as well as the solid substrate boundary cells.

\subsection{Performance Considerations}

Since the streaming and collision steps can be computed locally on each cell, LB methods can easily be parallelized. Therefore, the described approach has been implemented using the GPU-acceleration framework CUDA by Nvidia (Ref 36). All simulations have been carried out on the system described in section 5.3, which contains two GPUs to support single droplet simulations-one GeForce GTX Titan main stream card and one Nvidia Tesla K20c dedicated computation card. Throughout the coating simulations performed, the average computation time for one droplet, including all overhead like job- and heightmap-loading from file, was $15.2 \mathrm{~s}$ for the Tesla card and $10.3 \mathrm{~s}$ using the GTX Titan. Since the microstructure build-up simulation always processes multiple splats in parallel and may launch as many droplet simulations simultaneously as GPUs are available, one splat can be computed every $6.4 \mathrm{~s}$ yielding $\sim 564$ splats per hour.

Another key factor to increase the performance to this level was the separation of the significant amount of iterations needed to stabilize and smooth the particle distribution function $f$ after the insertion of the spherical droplet from the impact simulation. To accomplish this, after the creation of the spherical droplet within the simulation volume, 500 iterations are performed without applying any external forces to smooth the PDF, and the whole PDF for all cells is saved to file (see Fig. 3). For every following simulation with identical domain size, this precomputed PDF is loaded at the beginning. Small fluctuations in the PDF caused by the subsequent insertion of the boundary cells only affect the gaseous phase and are negligible. This approach removes the need to perform smoothing iterations in every run of the single-particle simulation. Smoothing iterations are computed only once for each volume size. Thereby, for a simulation with
12,000 splats, precomputed PDFs could be used in slightly more than $98.5 \%$ of the simulations.

\section{Coating Build-Up Simulation}

The coating build-up simulation is based on the idea that, for a given single-particle simulation method and configuration, the shape of a splat depends primarily on the shape of the underground at the impact location and that similar underground shapes result in similar splat shapes. This implies that splat shapes may be re-used for similar impact conditions and are allowed to introduce slight errors if they do not fit perfectly. A similar assumption has been made by Mostaghimi et al. who repeatedly placed one of four different splats solely based on the distance to the next closest splat (Ref 24). In contrast, in our approach, each splat is chosen based on the detailed heightmap-profile already present at its randomly computed impact position. Due to the multitude of possible underground shapes, the coating simulation benefits from high numbers of splats to choose from, although the introduced error by choosing nonideal splats of course decreases more rapidly with the first few splats in the database. With a certain number of splat shapes available in the simulation database, the computation time required to find and insert the best candidate becomes non-negligible. This is especially the case compared to the relatively short computation time for the described single splat simulation, but in principle holds for more sophisticated and slower simulations as well. Thus, three fast filters that pre-select a few candidate splats from the database have been designed and compared with each other, as well as with the optimal solution.

\subsection{Simulation Overview}

The microstructure simulation uses a voxel volume $V(\boldsymbol{x})$ with $\boldsymbol{x}=(x, y, z)^{\mathrm{T}} \in Z^{3}$ to represent the computed coating, where each $V(\boldsymbol{x}) \in[0,1]$ is the filled fraction of the voxel at $\boldsymbol{x}$. Initially, $V(\boldsymbol{x})=0$ for all voxels except for the solid substrate at the lower boundary of the volume $(V(\boldsymbol{x})=1)$. Using a voxel volume easily integrates with the droplet simulation lattice, which is assumed to have the same spacing. The volume fraction of a cell in the LB model can be transferred to the global volume $V$ by mapping $\rho(\boldsymbol{x}) \in\left[\rho_{\min }, \rho_{\max }\right]$ to $[0,1]$. Furthermore, the simulation tracks the current global heightmap of the simulated microstructure, given by the $z$-coordinate of the topmost solid voxel at every column $(x, y)$ :

$H_{V}(x, y)=\max (z \mid V(x, y, z) \geq 0.5)$.

Technically, $H_{V}$ changes with the insertion of new splats into $V$ and therefore is time dependent. However, to enhance readability, the time parameter is omitted here and we always refer to the latest state.

In addition to the global volume, the simulation makes use of a database of splats that have already been simu-

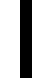



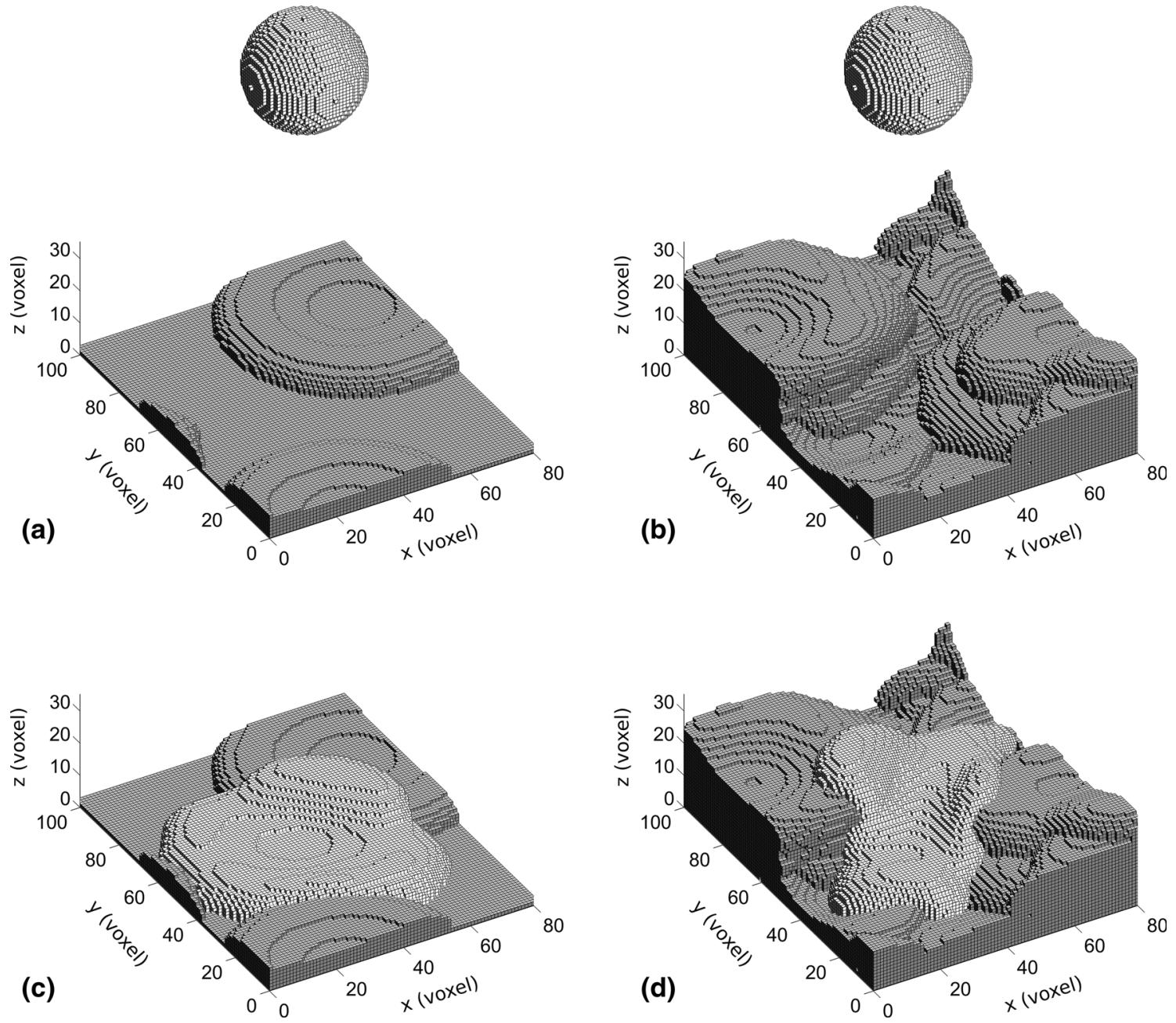

Fig. 2 Two-example configurations of the single-particle simulation. (a) and (b): Initial states showing the loaded heightmap profile and the initial position of the spherical droplet. (c) and (d): Resulting drop shapes on the profile after the impact and solidification
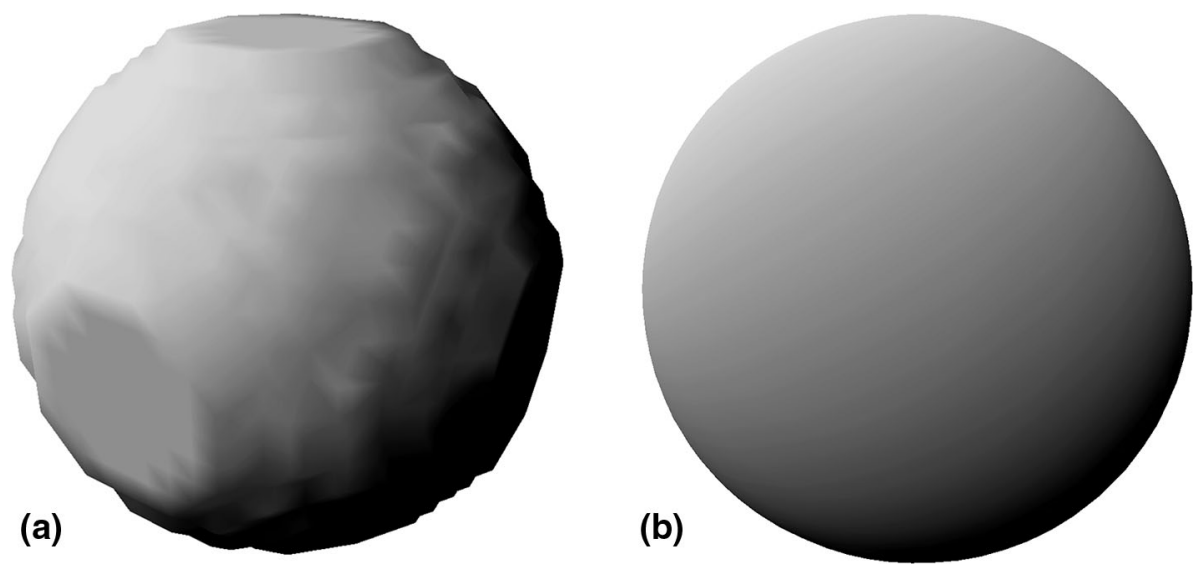

Fig. 3 Initial, edged shape of the spherical droplet (a) and smooth droplet after 500 iterations (b)

lated. This database consists of two files for each splat $i$. The first file contains the density field $\rho_{i}(\boldsymbol{x})=\rho_{i}\left(\boldsymbol{x}, t_{\mathrm{end}}\right)$ resulting from the droplet simulation. The second file contains the heightmap $H_{i}(x, y)$ representing the boundary that was used in the drop simulation as described in section 3.2. In the following, 
$S_{i}=\left\{\boldsymbol{x}=(x, y, z) \mid\left(\rho_{i}(\boldsymbol{x}) \geq\left(\rho_{\max }+\rho_{\min }\right) / 2\right) \wedge z>H_{i}(x, y)\right\}$

denotes the set of all solid voxels/cells of splat $i$. By default, the exact impact point of the particle that resulted in splat $i$ is at the center of the saved heightmap at $H_{i}\left(L_{x}\right)$ $\left.2, L_{y} / 2\right)$. In the database, all splats are sorted according to their ascending inner coverage radius $r_{\mathrm{c}}\left(H_{i}\right)$, which is defined as the radius of the largest circle centered at $\left(L_{x}\right)$ $\left.2, L_{y} / 2\right)$ that fits into the orthogonal projection $\Omega\left(S_{i}\right)$ of $S_{i}$ into the $(x, y)$-plane. This order is advantageous for the prefilters described later. Upon starting the coating buildup the following steps are performed:

(1) Randomly generate the requested number of impact points $\boldsymbol{p}_{j}=\left(x_{j}, y_{j}\right)$ uniformly distributed in the $(x, y)$ plane of $V$ and add them to a processing queue $Q$.

(2) Initialize $N$ worker threads $T_{k}$ to process impact points in parallel.

(3) For all idle workers $T_{k}$ assign the next $\boldsymbol{p}_{j}$ to $T_{k}$ and remove it from $Q$.

Each worker thread then inserts a new splat at position $\boldsymbol{p}_{j}$ on top of the current microstructure or, to be more precise, at $\left(\boldsymbol{p}_{j}, H_{V}\left(\boldsymbol{p}_{j}\right)\right)$. Specifically, the following steps are performed for splat $j$ :

(1) Wait until all impact positions $\boldsymbol{p}_{k}$ with $k<j$ and $\operatorname{dist}\left(\boldsymbol{p}_{j}, \boldsymbol{p}_{k}\right)<d_{\text {thresh }}$ are processed.

(2) Extract the local heightmap template $H_{T}(x, y)$ centered at $\boldsymbol{p}_{j}$ of size $L_{x} \times L_{y}$ from $H_{V}$.

(3) Search the database for candidate splats $i$ with similar heightmaps $H_{i}$ using one of the fast prefiltering methods from section 4.2.

(4) For all candidate splats $i$ perform an exact comparison of $H_{i}$ and $H_{T}$ to find the best matching underground $H_{i}^{*}$ for the current situation by means of the sum of squared errors (SSE).

(5) If the deviation $\operatorname{SSE}_{\min }\left(H_{i}^{*}, H_{T}\right)$ between $H_{i}^{*}$ and $H_{T}$ is smaller than a predefined threshold $\epsilon$, load the splat density field resulting from the droplet simulation with the underground $H_{i}^{*}$ into the final density field $\rho^{*}$.Otherwise start a new droplet simulation using $H_{T}$ as boundary and load the resulting density field into $\rho^{*}$.

(6) Insert $\rho^{*}$ into the microstructure $V$ at $\left(\boldsymbol{p}_{j}, H_{V}\left(\boldsymbol{p}_{j}\right)\right)$ as described in section 4.3.

Here, step 1 ensures that all computed impact positions are processed in the correct order and splats for different impact positions that may touch or overlap each other are not computed simultaneously. Most importantly, while one worker thread waits for a droplet simulation to finish, other splat impacts that are farther away from each other than $d_{\text {thresh }}$ continue to be processed in parallel. Steps 2-6 are illustrated in Fig. 4. For each precomputed impact position of a new splat the sur- rounding underground is extracted from the global voxel volume by means of the local heightmap $H_{T}$ which is then compared to all heightmaps in the database to find a similar impact situation. If a sufficiently similar heightmap is found the according splat can be inserted, otherwise a new droplet simulation is initiated and the resulting splat shape is inserted. For the simulation of a droplet, the local heightmap $H_{T}$ is saved into a file, a jobdescription file referring to this boundary description is created and the droplet simulation process is started as described in section 3 .

\subsection{Choosing a Splat}

For any given template heightmap $H_{T}$ modeling the surface patch around the impact point, it can be assumed without loss of generality that the particle always impacts perpendicular to the $(x, y)$-plane of the simulation volume, i.e., its velocity vector points along $(0,0$, $-z$ ), because any off-normal impact could be equally described using a surface patch rotated about the impact angle. Therefore, a surface patch found in the database can be considered a good match if it is similar to $H_{T}$ for at least one rotation $\phi$ in the $(x, y)$-plane about the impact point.

4.2.1 Exact Error. The exact minimum error between a template heightmap $H_{T}$ and a heightmap $H_{i}$ from the database is computed by the sum of squared errors minimized for all rotation angles $\phi$ :

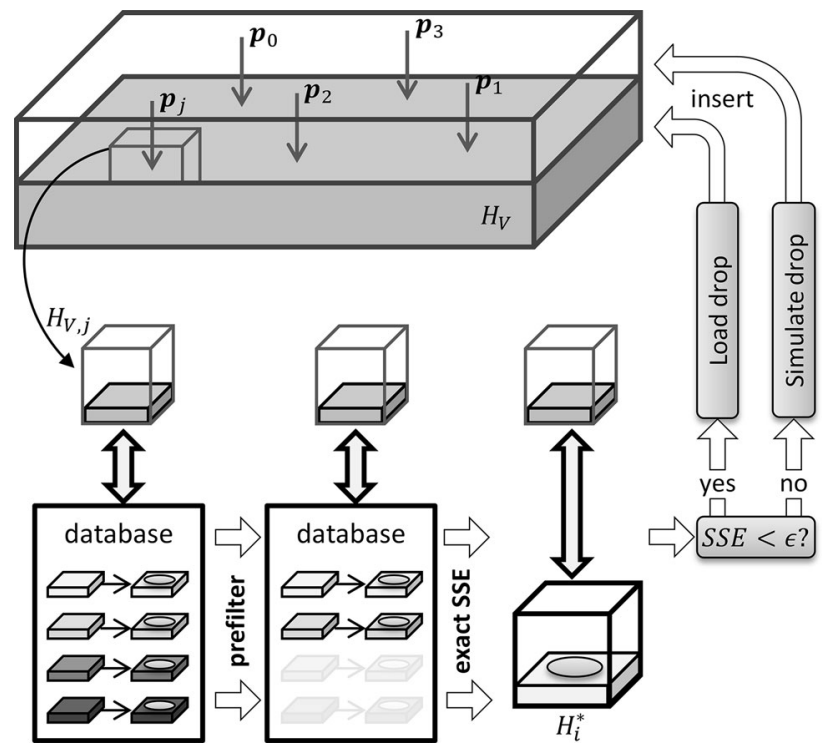

Fig. 4 Schematic overview of the sequential splat insertion process. Based on a heightmap extracted from the impact position $\boldsymbol{p}_{j}$, the best matching heightmap $H_{i}^{*}$ is determined by the two-step scheme using prefiltering and exact error computations. Only if $H_{i}^{*}$ meets the error threshold $\varepsilon$, the splat is inserted; otherwise a new droplet simulation is run 


$$
\begin{aligned}
\operatorname{SSE}_{\min }\left(H_{T}, H_{i}\right)= & \min _{\phi}\left[\sum _ { ( x , y ) \in \Omega ( S _ { i } ) } \left(H_{i}(x, y)-H_{T}\left(x_{\phi}, y_{\phi}\right)\right.\right. \\
& \left.\left.+\delta_{z}\left(H_{i}, H_{T}\right)\right)^{2}\right] .
\end{aligned}
$$

Therein, the evaluation is restricted to the area $\Omega\left(S_{i}\right)$ actually covered by $S_{i}$ and $x_{\phi}, y_{\phi}$ denote the coordinates $x, y$ rotated by $\phi$ about the impact point at the center of the heightmaps. The constant term $\delta_{z}$ offsets $H_{i}$ to match the average height of $H_{T}$ within $\Omega\left(S_{i}\right)$. For the analysis presented in section 5 , the more intuitive minimal absolute volume error is defined accordingly:

$$
\begin{aligned}
E_{\min }\left(H_{T}, H_{i}\right)= & \min _{\phi}\left[\sum_{(x, y) \in \Omega\left(S_{i}\right)} \mid H_{i}(x, y)-H_{T}\left(x_{\phi}, y_{\phi}\right)\right. \\
& \left.+\delta_{z}\left(H_{i}, H_{T}\right) \mid\right] .
\end{aligned}
$$

In practice, to compute $\mathrm{SSE}_{\min }$, the sum of squared errors must be evaluated for several rotations $k \cdot \delta \phi$. For the simulations performed $\delta \phi \approx 2.2^{\circ}$ was chosen, because for this value a splat with average diameter is rotated at least one voxel at its outer boundary and smaller values for $\delta \phi$ are insignificant. Thus, the computation of $\mathrm{SSE}_{\min }$ for a single $H_{i}$ from the database requires the evaluation of 164 rotations, resulting in a substantial computation time per template heightmap to be compared to all splats in the database. With the use of a suited filter that can be evaluated more quickly the search can be narrowed to a fraction of the database. For this purpose, different filters have been implemented and tested.
It can be seen that $O_{\mathrm{H}}$ divides the range $[0,2 \pi)$ of all possible directions of the gradient into equally sized intervals $0, \ldots, N-1$. The last code, $N$, is a special code denoting a zero gradient occurring in flat areas of $H$, which has no orientation. $\Psi_{H}$ is then computed as the histogram of orientation codes

$\Psi_{H}(k)=\sum_{(x, y) \in R_{\mathrm{c}}(H)} \delta\left(O_{H}(x, y)-k\right)$

for $k=0, \ldots, N$ and within the inner coverage area

$R_{\mathrm{c}}(H)=\left\{(x, y) \mid \sqrt{x^{2}+y^{2}} \leq r_{\mathrm{c}}(H)\right\}$

and with $\delta(\cdot)$ being the Kronecker delta function. So, basically, the orientation code histogram uses the frequency of occurrence of $N$ different slope directions to describe the heightmap. For an estimated comparison of two heightmaps, their histograms $\Psi_{H_{i}}$ and $\Psi_{H_{T}}$ can be compared. A key observation for the efficient comparison is the fact that if $H$ is rotated by $\phi=2 \pi / N$ all orientation codes are also rotated by $\phi$ and the histogram of the rotated heightmap $H^{\phi}$ can simply be computed by a cyclic shift of the first $N$ histogram values by $1: \Psi_{H^{\phi}}(k)=$ $\Psi_{H}((k-1) \bmod N)$. Thus, all rotations $\phi=k \cdot 2 \pi / N$ of $H$ can be checked by $N$ simple shift operations. Because the computation of $\Psi$ has been restricted to the circular area $R_{\text {c }}$, each shifted histogram refers to exactly the same area. For all splats $H_{i}$ in the database, $\Psi_{H_{i}}$ is precomputed. To compare $H_{T}$ with all database entries, $\Psi_{H_{T}}$ has to be recomputed every time the inner coverage radius $r_{\mathrm{c}}\left(H_{i}\right)$ of the splat to be compared against changes. Since the database is sorted by $r_{\mathrm{c}}\left(H_{i}\right)$ and the radius is considered an integer, a recomputation is only required a few times $(<20)$ per template $H_{T}$. To compute a correlation value $C_{\mathrm{OH}}$ for two histograms with relative rotation $\phi=k \cdot 2 \pi / n$, intersections (minima) of histogram bins are considered:

$C_{\mathrm{OH}}\left(H_{i}, H_{T}, k\right)=\frac{\min \left\{\Psi_{\mathrm{H}_{\mathrm{i}}}(\mathrm{N}), \Psi_{\mathrm{H}_{\mathrm{T}}}(\mathrm{N})\right\}+\sum_{\mathrm{l}=0}^{\mathrm{N}-1} \min \left\{\Psi_{\mathrm{H}_{\mathrm{i}}}((1+\mathrm{k}) \bmod \mathrm{N}), \Psi_{\mathrm{H}_{\mathrm{T}}}(\mathrm{l})\right\}}{\sum_{l=0}^{N} \Psi_{H_{i}}(l)}$.

4.2.2 Orientation Codes. The first filter is based on the idea of orientation code histograms described in the context of automatic image segmentation by Na et al. (Ref 37). The orientation code histogram $\Psi_{H}$ of a heightmap $H$ can be considered a feature vector characterizing $H$ that is used for a fast comparison. First of all, the orientation code $O_{\mathrm{H}}(x, y)$ is defined as the discretization of the angular direction of the gradient $\nabla H(x, y)=\left(\Delta H_{x}, \Delta H_{y}\right)^{\mathrm{T}}$ :

$$
\begin{aligned}
& O_{H}(x, y) \\
& = \begin{cases}\frac{N-1}{2 \pi} \cdot\left[a \tan 2\left(\Delta H_{y}, \Delta H_{x}\right)+\pi\right] \in[0, N-1], & \left|\Delta H_{x}\right|+\left|\Delta H_{y}\right|>0 \\
N, & \text { else. }\end{cases}
\end{aligned}
$$

For all histogram bins, the number of discrete positions $(x, y)$ in $H_{i}$ and $H_{T}$ sharing a gradient direction are summed and normalized by the total number of considered positions. Thus, $0 \leq C_{\mathrm{OH}} \leq 1$ and larger values for $C_{\mathrm{OH}}$ indicate better matches. The best correlation value is then identified over all $N$ rotations

$C_{\mathrm{OH}}\left(H_{i}, H_{T}\right)=\max _{k} C_{\mathrm{OH}}\left(H_{i}, H_{T}, k\right)$

and the corresponding rotation $k_{\mathrm{opt}}$ can be used to estimate the rotation of $H_{i}$. Once all underground shapes from the database have been compared, the exact error $\mathrm{SSE}_{\min }\left(H_{T}, H_{i}\right)$ is computed for the $n$ best candidates or 
all candidates exceeding a minimal $C_{\mathrm{OH}}$. Within the exact error calculation the rotation estimate $k_{\text {opt }}$ is used to restrict the tested rotations $\phi$ to an interval of size $2 \pi / N$ centered at $k_{\text {opt }} \cdot 2 \pi / N$.

4.2.3 Orientation Locations. One drawback of the orientation codes is that they solely rely on the occurrence frequency of different gradient orientations within the circular computation domain $R_{\mathrm{c}}$, but do not include any location information as to where in relation to the impact point the different gradients can be found. Therefore, another prefilter has been designed, relying on the average position $\Phi_{H_{i}}(k)$ of the gradient positions $(x, y)$ belonging to each histogram bin $\Psi_{H_{i}}(k)$. Normalizing the position to the inner coverage radius $r_{\mathrm{c}}\left(H_{i}\right)$, the average gradient position for bin $k$ is

$\Phi_{H_{i}}(k)=\frac{1}{r_{\mathrm{c}}\left(H_{i}\right) \Psi_{H_{i}}(k)} \sum_{(x, y) \in R_{c}\left(H_{i}\right)} \delta\left(O_{H}(x, y)-k\right) \cdot(x, y)^{\mathrm{T}}$

for $\Psi_{\mathrm{H}_{\mathrm{i}}}(k)>0$ and $\Phi_{H_{i}}(k)=(0,0)^{\mathrm{T}}$ otherwise. To compare $\Phi_{H_{i}}$ with $\Phi_{H_{T}}$ considering a rotation $\phi=k \cdot 2 \pi / N$, in addition to shifting $k$, the locations $\Phi_{H_{i}}(k)$ have to be rotated around the origin using a rotation $\boldsymbol{R}_{\phi}$. As for the orientation codes, a correlation value in $[0,1]$ is obtained by

$C_{O L}\left(H_{i}, H_{T}, k\right)=1-\frac{1}{2 N} \sum_{l=0}^{N-1}\left\|\boldsymbol{R}_{\phi} \Phi H_{i}((l+k) N)-\Phi_{H_{T}}(l)_{2}\right\|$,

where, again, larger values indicate a better correlation. In correspondence to Eq 19, the optimal correlation $C_{\mathrm{OL}}\left(H_{i}, H_{T}\right)$ and the according rotation are found by maximizing $C_{\mathrm{OL}}\left(H_{i}, H_{T}, k\right)$ for all $k$.

4.2.4 Cyclic Ring Samples. A third prefilter approach has been developed that combines a reduced version of the exact error SSE formulation with the efficient rotation computation found in the orientation gradient-based algorithms. For this, the SSE is evaluated only at $Q N$ specific points equally distributed on $Q$ rings with radii $q_{\text {r }}$ around the impact point $\boldsymbol{p}=0$ :

$$
\begin{gathered}
\Upsilon_{H}(r, k)=H\left(q_{r} \cdot \cos \left(k \cdot \frac{2 \pi}{N}\right), q_{r} \cdot \sin \left(k \cdot \frac{2 \pi}{N}\right)\right), \\
1 \leq r \leq Q, \quad 0 \leq k \leq N-1
\end{gathered}
$$

Therein, the $q_{\mathrm{r}}$ have been chosen to fit into most splat areas $\Omega\left(S_{i}\right)$. Samples that are outside this area are ignored in the computations. Since the heightmap samples $\Upsilon$ are taken from equally spaced angles around the impact point, $\Upsilon$ can rotated by shifting $k$ as before. The error for a given rotation $k$ is

$$
\begin{aligned}
E_{\mathrm{CRS}}\left(H_{i}, H_{T}, k\right)= & \frac{1}{Q N} \sum_{r=1}^{Q} \sum_{l=0}^{N-1}\left(\Upsilon_{H_{i}}(r,(l+k) \bmod N)\right. \\
& \left.-\Upsilon_{H_{T}}(r, l)+\delta_{z}\left(H_{i}, H_{T}\right)\right)^{2} \quad(\mathrm{Eq}
\end{aligned}
$$

with $\delta_{Z}$ denoting the average height offset. From this, the correlation value increasing with better matching heightmaps is defined as

$$
C_{\mathrm{CRS}}\left(H_{i}, H_{T}, k\right)=\frac{1}{\sqrt{1+E_{\mathrm{CRS}}\left(H_{i}, H_{T}, k\right)}} .
$$

As with the other filters the optimum $C_{\mathrm{CRS}}\left(H_{i}, H_{T}\right)$ and $k_{\text {opt }}$ are found by maximizing $C_{\mathrm{CRS}}\left(H_{i}, H_{T}, k\right)$ for all $k$.

\subsection{Splat Insertion Algorithm}

Once the employed prefilter has limited the database to a relatively small number of candidate splats, the exact error is computed for all of them to find the single best splat and a corresponding best rotation $\phi_{\text {opt }}$. If the best splat found in the database does not satisfy the maximum absolute error criterion $\epsilon$, a new splat is simulated. The insertion of the splat into the global simulation volume $V$ is the same for both simulated splats or splats loaded from the database, however, for simulated ones no rotation is required, i.e., $\phi_{\text {opt }}=0$.

For the insertion of a splat, its density distribution $\rho_{i}(\boldsymbol{x})$ is rotated within the $x, y$-plane according to the computed optimal angle $\phi_{\text {opt }}$ and translated to the impact point $(x, y)$-coordinates. Thereafter, to align the $x$ - and $y$-grid axes of $\rho_{i}$ and $V, \rho_{i}$ is transferred to a temporary voxel grid $V^{*}$, whose axes are parallel to the axes of the target volume $V$, by resampling $\rho_{i}$ with 25 samples per voxel in $V^{*}$. This establishes a one-to-one correspondence of voxel columns in $V^{*}$ and $V$, i.e., the $x$ - and $y$-coordinates for each voxel in $V^{*}$ are already final.

For the alignment in the $z$-direction, $\rho_{i}$ still needs to be translated by $\delta_{z}$, which is done in two steps. First, $\rho_{i}$ is translated by the integer part $\delta_{z}+0.5$ of $\delta_{z}$ keeping the resulting voxel grid perfectly aligned with the grid of $V$ in the $z$-direction. To retain precise isosurface boundaries of the splat represented by the density field, the fractional part $\delta_{z}^{*} \in[-0.5,0.5]$ of $\delta_{z}$ is taken into account by a linear interpolation of densities between neighboring voxels according to $\delta_{z}^{*}$ as shown in Fig. 5. This results in a second temporary volume $V^{* *}$, where each voxel position in $V^{* *}$ exactly corresponds to a voxel in $V$.

The splat is then copied to the global volume $V$ by keeping the maximum of densities of two corresponding cells, i.e. $V_{\text {new }}(\boldsymbol{x})=\max \left(V_{\text {old }}(\boldsymbol{x}), V^{* *}(\boldsymbol{x})\right)$. This allows airvoxels to be replaced by solid voxels belonging to the new splat, but no solid voxels may be removed. All air-voxels converted to solid voxels are labeled with the id number of the inserted splat to be able to track all splats within $V$. Finally, the global heightmap $H_{V}$ is updated and, for visualization purposes, the newly inserted splat volume's isosurface is triangulated using a marching cubes algorithm.

It should be clear that the insertion method exactly resembles the single-particle simulation results only if the splat has been specifically simulated for the exact underground patch at hand. Otherwise, mass may be lost during insertion since there is nearly always a non-zero discrepancy between the current and the best candidate's heightmap. However, by carefully selecting the maximum allowed error $\epsilon$, the maximum percentage of mass lost per splat can be controlled. 


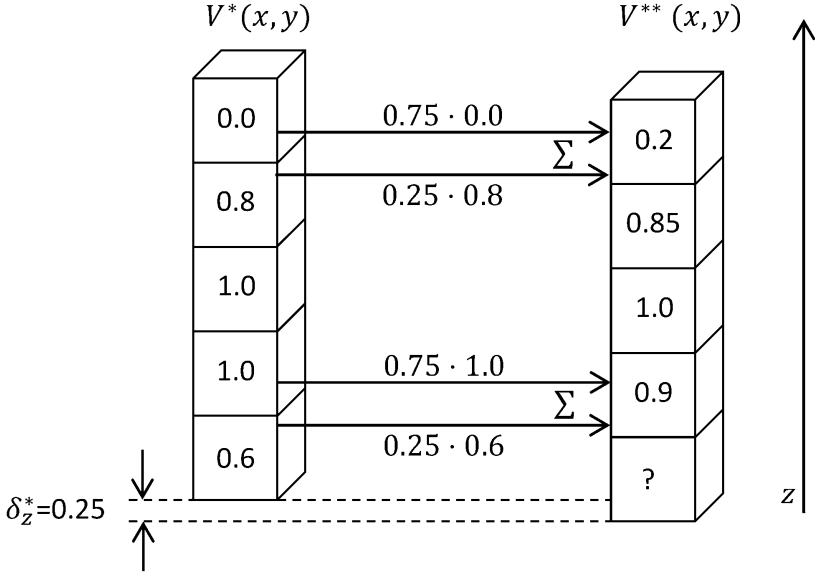

Fig. 5 Subvoxel-accurate vertical alignment of a voxel column during splat insertion. Density values in the new volume $V^{* *}$ are linearly interpolated according to a fractional part $\delta_{z}^{*}=0.25$ of the required translation

\section{Results}

Several simulations have been performed to assess and compare the performance of the prefilters, to visually analyze splat selection and insertion as well as to measure computation times.

\subsection{Prefilter Performance}

The objective of the prefiltering approaches presented in section 4.2 is to reduce the number of expensive computations of the exact heightmap-to-heightmap errors. Therefore, a good prefilter should be able to rule out a lot of splats from the database while still finding the best available splat geometries. To compare the performance of the prefilters, a splat database with 10,793 splats has been created by a single run of the microstructure simulation, creating a large microstructure volume (see Fig. 6). The database has then been divided into five chunks containing 2000 splats and their respective boundary heightmaps. Within a chunk, each heightmap has been compared to every other heightmap with respect to the exact squared and absolute error as well as with respect to the three correlation values $C_{\mathrm{OH}}, C_{\mathrm{OL}}$, and $C_{\mathrm{CRS}}$. With this data, a definitive ranking of candidate splats for a given heightmap could be established using the exact absolute error values. The reasons for the division into chunks were to reduce computation time for the error computations and to check whether the results are different for splats at the beginning versus the end of the simulation run. Indeed, it has been found that, due to the lower variability within the heightmaps close to the initially flat substrate, the best achievable exact error for each heightmap is significantly lower for the first 2000 splats than for locations farther away from the substrate. No significant differences in the achievable error values were found for the splat numbers above 2000.

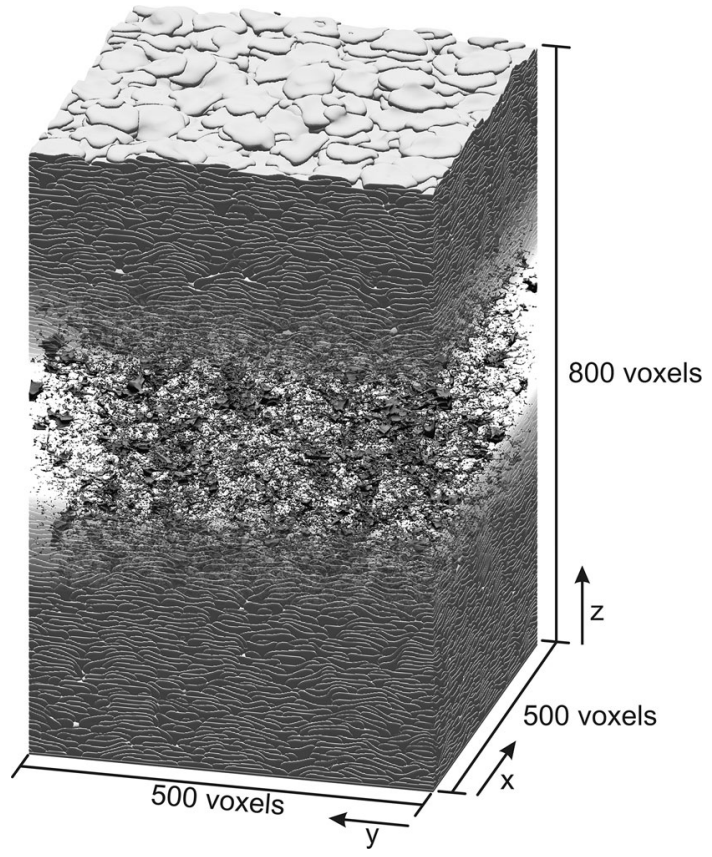

Fig. 6 Simulated microstructure volume containing 9737 splats with an $\mathrm{x}$-ray view through the pore network in the middle. For this volume, all splats have been simulated and none are inserted from the database

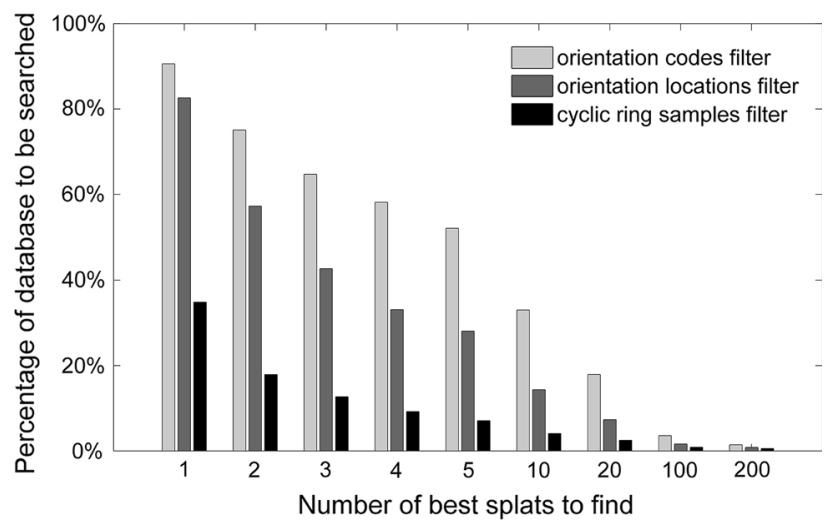

Fig. 7 Percentage of the number of splats in the database that need to be analyzed using the exact error computation to find one of the $N(1, \ldots, 200)$ best splats with a probability of at least $90 \%$ after one of the three prefilters has been applied

To quantify the performance of each filter, two questions were investigated:

(1) How many of the best candidates found with the filter must be considered for exact comparisons to find one of the $N$ real best solutions with a chance of at least $90 \%$ ?

(2) What is the expected absolute error in relation to the optimal candidate's error when considering $x \%$ of the best splats from the candidate ranking of the filter?

The answer to the first question is given in Fig. 7, showing the number of splats to be considered for exact 


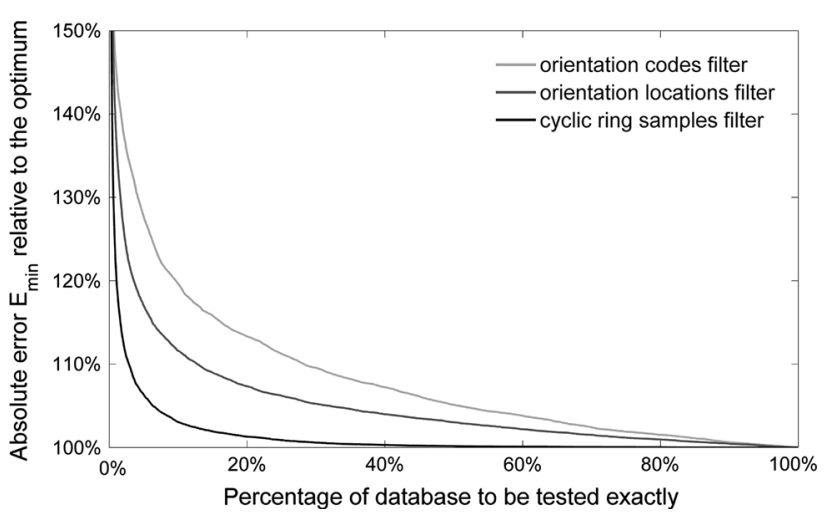

Fig. 8 Expected error of the chosen splat compared to the error value of the optimal splat depending on the percentage of candidate splats selected by one of the prefilters. The more candidates are a priori discarded, the higher the expected error

error-testing as a percentage of the total number of splats. For example, to find one of the five best splats with a $90 \%$ chance using the cyclic ring samples filter, costly exact comparisons have to be performed for only $7.1 \%$ of the splats in the database. In other words, $92.9 \%$ of the candidates are ruled out. Obviously, the cyclic ring sample filter performs best, followed by the orientation locations filter and the orientation codes filter performs worst.

The expected absolute error in relation to the absolute error of the optimal candidate splat is plotted in Fig. 8 for each filter method. Again, the performance ranking is the same with the cyclic ring samples filter performing best. Continuing the above example looking for one of the five best solutions, and therefore checking only $7.1 \%$ of the database exactly, the average achieved error is about $104.5 \%$ of the error for the best possible solution. For this amount of exact comparisons, the location orientation filter reaches only a relative error of $114 \%$ and the orientation codes filter $123 \%$.

Possible reasons for this outcome may be that the computation of $C_{\mathrm{CRS}}$ is by design very similar to the computation of the exact error or that the distributions of orientation gradients are too similar to each other due to the limited number of different gradients occurring at voxel boundaries.

\subsection{Visual Analysis}

Figure 6 shows a computed microstructure volume consisting of 9373 splats that have all been simulated independently without using splats already contained in the database. The values of the relevant parameters of the splat simulation mentioned in section 3 have been chosen to yield pancake-type splat shapes visually agreeing to the ones observed in other experiments (Ref 9, 35). In the middle of the volume, the inner structure of the pore network created from gaseous voxels in between the splats is revealed. The total closed porosity of the volume is $6.9 \%$. It can be seen at the volume boundaries that all splats are tightly packed and that their shapes are clearly

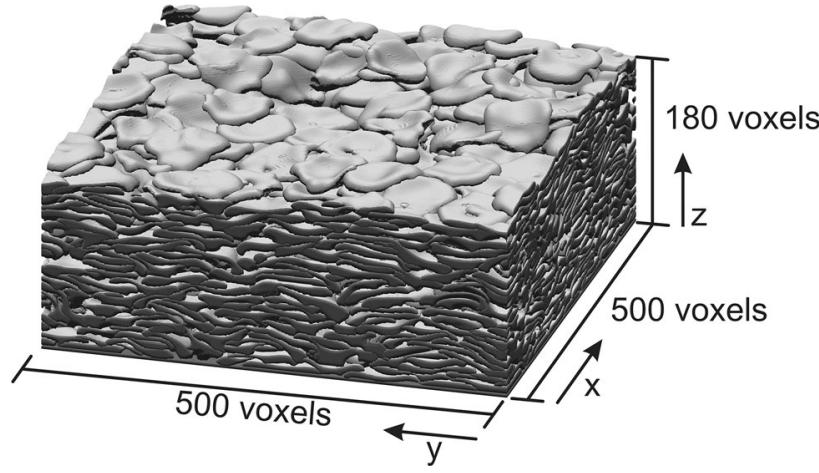

Fig. 9 Computed volume with about 1300 splats inserted only from the database. This approach creates irregular-shaped steep ridges and other artifacts making it difficult to find good matching splats, which results in a very high porosity

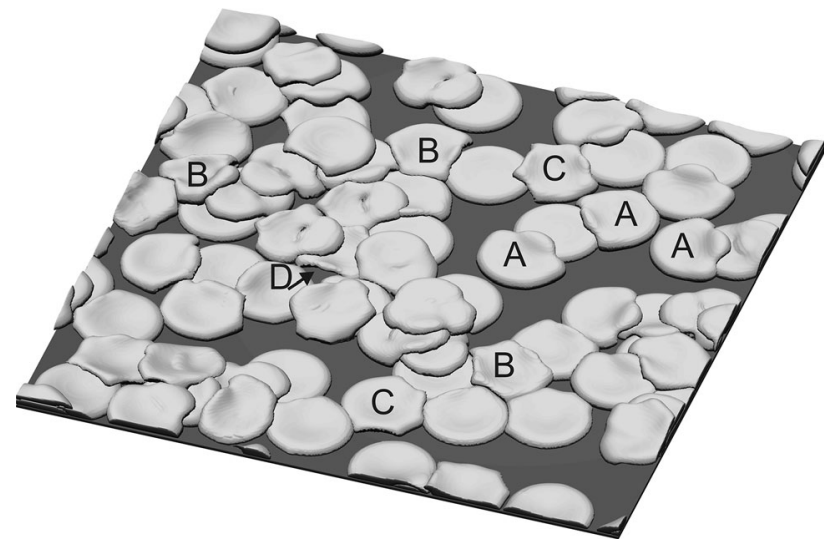

Fig. 10 The first splats in a new volume, all inserted from the database. Due to the lower geometric diversity near the substrate, good matches can easily be found for single overlap situations (A) as well as more complicated undergrounds (B). However, since fitting error computations are limited to the splat coverage area $\Omega\left(S_{i}\right)$, some splats show illogical gaps where their spreading was hindered by other splats in their original impact situation (C). An example for a vertical drop introduced by a non-fitting splat is pointed out by (D)

influenced by the neighboring splats. Most of the splat geometries can be described as pancake- or disk-like without strong fingering but some steep slopes (Ref 7); however, there are also non-pancake-like splats, whose shapes are influenced by cavities in the underground that were hit.

When including splats from the database using the search and insertion method described, the subsequent insertion of imperfectly matching splats from the database on top of each other leads to the development of a more diverse underground including frequent and higher vertical drops at splat boundaries due to unfilled voids below the border of the splats. Because of these untypical features, throughout the course of the simulation, it becomes more difficult to find well matching splats in the database 


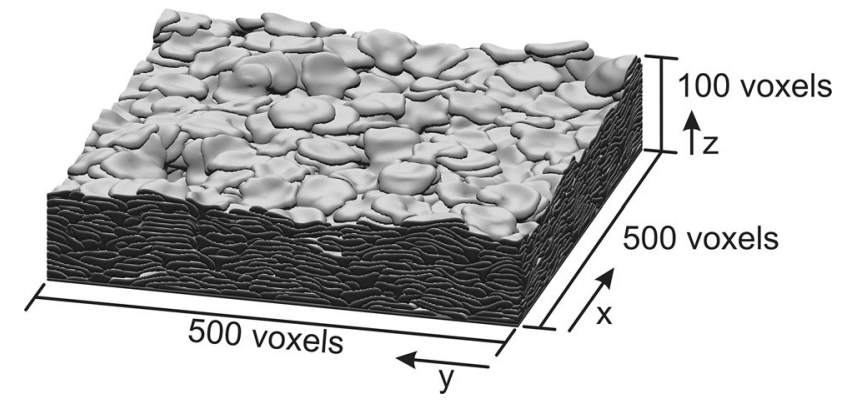

Fig. 11 Computed volume with about 1300 splats, whereof $\approx 75 \%$ have been simulated and $\approx 25 \%$ have been inserted from the database. As opposed to the volume in Fig. 9, possibly introduced artifacts due to improper fitting splats are smoothed out by simulated splats

and the fitting quality of splats decreases significantly while the porosity increases. In the extreme case of a microstructure build-up process using only database splats without employing a maximum error threshold $\epsilon$ results in an unrealistic volume as shown in Fig. 9. Nevertheless, at the beginning of the simulation, most of the splats found in the database visually match the underground well as depicted in Fig. 10.

As opposed to this, the microstructure displayed in Fig. 11 has been computed with a moderate error limit $\epsilon$, causing about $25 \%$ of the inserted splats to be selected from the database. For this, the cyclic ring samples filter has been employed to discard a careful $60 \%$ of the database entries a priori. In this case, the fewer introduced gaps from database originating splats are smoothed out by the simulated ones. The final porosity is $9.2 \%$.

\subsection{Overall Performance}

The performance of the simulation was measured on a high performance desktop workstation "Celsius R920 Power" by Fujitsu containing two Intel Xeon E5-2690 CPUs at $2.9 \mathrm{GHz}$ with 16 cores each, 64 GB DDR3 RAM and, for the GPU-powered single droplet simulations, a Nvidia GeForce GTX Titan (first generation) and a Nvidia Tesla k20c dedicated computation card. As already stated in section 3.4, with the two GPU devices a single droplet simulation requires $12.8 \mathrm{~s}$ on average, so a new drop is computed every $6.4 \mathrm{~s}$. The basis for all measurements is a database with 10,793 splats and all of the following timings refer to computations using only one of the 32 cores, since parallelization mainly occurs on a higher level by distributing several splat searches to different computation threads. Searching the best drop and its optimal rotation by employing only the exact error method, one drop search requires $158.5 \mathrm{~s}$, since 68.1 heightmap comparisons can be performed per second. For the three filters, the time required for the histogram comparisons are close to negligible $\left(\approx 3 \times 10^{-6} \mathrm{~s}\right)$. However, for the orientation code-based filters the histogram $\Psi_{H_{T}}$ of the template heightmap has to be recomputed once for each coverage radius $r_{\mathrm{c}}$ in the database-17 times for the database used. Therefore, the total time required to filter the whole database is $\approx 0.58 \mathrm{~ms}$ for the orientation code-based filters as opposed to only $0.066 \mathrm{~ms}$ for the cyclic ring samples, which do not require histogram recomputations. For the candidates found by prefiltering, exact error computations have to be performed for 12 rotations within the rotation angle range found by the prefilter. The total time to find the optimal splat using the cycling ring samples approach thus is

$T_{\mathrm{CRC}}(N)=0.066 \mathrm{~ms}+N \cdot 0.98 \mathrm{~ms}$,

where $N$ is the number of candidate splats left over by the filter. It can thus be concluded that the computation time of the prefilter itself is negligible. Since the filter may safely rule out at least $60-80 \%$ of the candidate splats, finding a well matching splat takes about 2-4 s per computation core.

\section{Summary and Conclusion}

In this paper, a comprehensive approach modeling the microstructure formation in thermal spraying by means of a coupled single-particle- and global microstructuresimulation has been presented. As of this coupling, single splats inserted into the microstructure are simulated in an underground-sensitive manner and large microstructure volumes with more than 10,000 splats were successfully computed.

Furthermore, the microstructure construction may be accelerated by re-using suitable splats that have already been computed and stored in a database. By re-using only splats that obey to a maximum error threshold $\epsilon$, the overall error introduced by imperfectly fitting splats can be fully controlled. With a database of about 10,000 splats and a reasonably chosen threshold $\epsilon$ a significant fraction of splats can be taken from the database thereby saving computational costs of single-particle simulations. This benefit is expected to further increase with the continuous growth of the database.

To find good matching splats, three different filters have been designed and thoroughly tested. From these filters, the cyclic ring samples filter performs best and is able to significantly speed up splat selection, while still finding good matches.

The coupled simulation approach works well and is easily applicable to other single-particle simulations as well, since the geometry-based interface is not very restrictive. The probability of finding well matching splats via the database approach depends on the variability of generated underground shapes and the size of the database. Especially for the use of single-particle simulations modeling more sophisticated fingering and splashing, a combination of the presented approach with already developed geometric fitting operations, like the filtering technique presented earlier in Ref 38, may be beneficial for the reduction of the error introduced by the splat insertion while maintaining the better part of the sensitivity of the splat shape to the underground. 


\section{Acknowledgments}

This research was funded by the German Research Foundation (DFG) as part of the collaborative research center 708 "3D-Surface Engineering of Tools for Sheet Metal Forming-Manufacturing, Modeling, Machining," subproject B1.

\section{References}

1. C.-J. Li and A. Ohmori, Relationships Between the Microstructure and Properties of Thermally Sprayed Deposits, J. Therm. Spray Technol., 2002, 11(3), p 365-374

2. R. Ghafouri-Azar, J. Mostaghimi, and S. Chandra, Modeling Development of Residual Stresses in Thermal Spray Coatings, Comput. Mater. Sci., 2006, 35(1), p 13-26

3. I.O. Golosnoy, A. Cipitria, and T.W. Clyne, Heat Transfer Through Plasma-Sprayed Thermal Barrier Coatings in Gas Turbines: a Review of Recent Work, J. Therm. Spray Technol., 2009, 18(5-6), p 809-821

4. A. Kulkarni, Z. Wang, T. Nakamura, S. Sampath, A. Goland, H. Herman, J. Allen, J. Ilavsky, G. Long, J. Frahm, and R.W. Steinbrech, Comprehensive Microstructural Characterization and Predictive Property Modeling of Plasma-Sprayed Zirconia Coatings, Acta Mater., 2003, 51(9), p 2457-2475

5. T. Inamuro, T. Ogata, S. Tajima, and N. Konishi, A Lattice Boltzmann Method for Incompressible Two-Phase Flows with Large Density Differences, J. Comput. Phys., 2004, 198(2), p 628644

6. P. Yuan and L. Schaefer, A Thermal Lattice Boltzmann TwoPhase Flow Model and Its Application to Heat Transfer Problems-Part 2. Integration and Validation, J. Fluids Eng., 2006, 128(1), p 151

7. P. Fauchais, M. Fukumoto, A. Vardelle, and M. Vardelle, Knowledge Concerning Splat Formation: An Invited Review, J. Therm. Spray Technol., 2004, 13(3), p 337-360

8. S. Chandra and P. Fauchais, Formation of Solid Splats During Thermal Spray Deposition, J. Therm. Spray Technol., 2009, 18(2), p 148-180

9. K. Yang, M. Liu, K. Zhou, and C. Deng, Recent Developments in the Research of Splat Formation Process in Thermal Spraying, $J$. Mater., 2013, 2013(2), p 1-14

10. O.P. Solonenko, V.V. Kudinov, A.V. Smirnov, A.N. Cherepanov, V.N. Popov, A.A. Mikhalchenko, and E.V. Kartaev, Micrometallurgy of Splats: Theory, Computer Simulation and Experiment, JSME Int. J. B, 2005, 48(3), p 366-380

11. Y. Tan, J.P. Longtin, and S. Sampath, Modeling Thermal Conductivity of Thermal Spray Coatings: Comparing Predictions to Experiments, J. Therm. Spray Technol., 2006, 15(4), p 545-552

12. F. Azarmi, T. Coyle, and J. Mostaghimi, Young's Modulus Measurement and Study of the Relationship Between Mechanical Properties and Microstructure of Air Plasma Sprayed Alloy 625, Surf. Coat. Technol., 2009, 203(8), p 1045-1054

13. B. Klusemann, R. Denzer, and B. Svendsen, MicrostructureBased Modeling of Residual Stresses in Wc-12Co-Sprayed Coatings, J. Therm. Spray Technol., 2012, 21(1), p 96-107

14. T. Wiederkehr, B. Klusemann, D. Gies, H. Müller, and B. Svendsen, An Image Morphing Method for 3D Reconstruction and Fe-Analysis of Pore Networks in Thermal Spray Coatings, Comput. Mater. Sci., 2010, 47(4), p 881-889

15. N. Chawla, V.V. Ganesh, and B. Wunsch, Three-Dimensional (3D) Microstructure Visualization and Finite Element Modeling of the Mechanical Behavior of Sic Particle Reinforced Aluminum Composites, Scripta Mater., 2004, 51(2), p 161-165

16. K. Bobzin, N. Kopp, T. Warda, and M. Öte, Determination of the Effective Properties of Thermal Spray Coatings Using 2D and 3D Models, J. Therm. Spray Technol., 2012, 21(6), p 1269-1277

17. W.E. Lorensen and H.E. Cline, Marching Cubes: a High Resolution 3D Surface Construction Algorithm, SIGGRAPH Comput. Graph., 1987, 21(4), p 163-169
18. T. Lewiner, H. Lopes, and A.W. Vieira, Efficient Implementation of Marching Cubes' Cases with Topological Guarantees, J. Graph. Tools, 2003, 8(2), p 1-15

19. K. Holmberg, A. Laukkanen, A. Ghabchi, M. Rombouts, E. Turunen, R. Waudby, T. Suhonen, K. Valtonen, and E. Sarlin, Computational Modelling Based Wear Resistance Analysis of Thick Composite Coatings, Tribol. Int., 2013, 72, p 13-30

20. J.-H. Qiao, R. Bolot, H. Liao, P. Bertrand, and C. Coddet, A 3D Finite-Difference Model for the Effective Thermal Conductivity of Thermal Barrier Coatings Produced by Plasma Spraying, Int. J. Therm. Sci., 2013, 65, p 120-126

21. Z. Wang, A. Kulkarni, S. Deshpande, T. Nakamura, and H. Herman, Effects of Pores and Interfaces on Effective Properties of Plasma Sprayed Zirconia Coatings, Acta Mater., 2003, 51(18), p 5319-5334

22. H. Singh, "Computer Simulations of Realistic Microstructures: Implications for Simulation-Based Materials Design," Dissertation, Georgia Institute of Technology, 2007

23. S. Cirolini, J.H. Harding, and G. Jacucci, Computer Simulation of Plasma-Sprayed Coatings I. Coating Deposition Model, Surf. Coat. Technol., 1991, 48(2), p 137-145

24. R. Ghafouri-Azar, J. Mostaghimi, S. Chandra, and M. Charmchi, A Stochastic Model to Simulate the Formation of a Thermal Spray Coating, J. Therm. Spray Technol., 2003, 12(1), p 53-69

25. J. Mostaghimi, S. Chandra, R. Ghafouri-Azar, and A. Dolatabadi, Modeling Thermal Spray Coating Processes: A Powerful Tool in Design and Optimization, Surf. Coat. Technol., 2003, 163164, p 1-11

26. S. Beauvais, V. Guipont, M. Jeandin, D. Jeulin, A. Robisson, and R. Saenger, Study of the Porosity in Plasma-Sprayed Alumina Through an Innovative Three-Dimensional Simulation of the Coating Buildup, Metall. Mater. Trans. A, 2008, 39(11), p 27112724

27. O.P. Solonenko, V.A. Blednov, and V.I. Iordan, Computer Design of Thermal Sprayed Metal Powder Coatings, Thermophys. Aeromech., 2011, 18(2), p 255-272

28. T. Opfermann, "Simulation der Kollision und Erstarrung von Tropfen auf Oberflächen mittels der Lattice-Boltzmann-Methode und CUDA," Diplomarbeit, TU Dortmund, German, 2010

29. P. Yuan and L. Schaefer, A Thermal Lattice Boltzmann TwoPhase Flow Model and Its Application to Heat Transfer Problems-Part 1. Theoretical Foundation, J. Fluids Eng., 2006, 128(1), p 142

30. J. Bao and L. Schaefer, Lattice Boltzmann Equation Model for Multi-Component Multi-phase Flow with High Density Ratios, Appl. Math. Model., 2013, 37(4), p 1860-1871

31. X. Shan and H. Chen, Lattice Boltzmann Model for Simulating Flows with Multiple Phases and Components, Phys. Rev. E, 1993, 47(3), p 1815-1819

32. X. Shan and H. Chen, Simulation of Nonideal Gases and LiquidGas Phase Transitions by the Lattice Boltzmann Equation, Phys. Rev. E, 1994, 49(4), p 2941-2948

33. P. Bhatnagar, E. Gross, and M. Krook, A Model for Collision Processes in Gases. I. Small Amplitude Processes in Charged and Neutral One-Component Systems, Phys. Rev., 1954, 94(3), p 511525

34. E.A. Semma, M. El Ganaoui, and R. Bennacer, Lattice Boltzmann Method for Melting/Solidification Problems, C. R. Mec., 2007, 335(5-6), p 295-303

35. M. Pasandideh-Fard, V. Pershin, S. Chandra, and J. Mostaghimi, Splat Shapes in a Thermal Spray Coating Process: Simulations and Experiments, J. Therm. Spray Technol., 2002, 11(2), p 206217

36. N. Wilt, The Cuda Handbook: A Comprehensive Guide to GPU Programming, 1st ed., Addison-Wesley, Upper Saddle River, 2013

37. Y. Na, S. Bingxia, X. Jia, and Z. Quanming, Orientation CodesBased Template Matching Method on Workpiece Detection, 2nd International Conference on Power Electronics and Intelligent Transportation System (Peits) (Shenzhen, China), 2009, p 70-73

38. A. Kout, T. Wiederkehr, and H. Müller, Efficient Stochastic Simulation of Thermal Spray Processes, Surf. Coat. Technol., 2009, 203(12), p 1580-1595 